\title{
THE HIGHER-ORDER MATCHING POLYNOMIAL OF A GRAPH
}

\author{
OSWALDO ARAUJO, MARIO ESTRADA, \\ DANIEL A. MORALES, AND JUAN RADA
}

Received 27 April 2004 and in revised form 1 March 2005

Given a graph $G$ with $n$ vertices, let $p(G, j)$ denote the number of ways $j$ mutually nonincident edges can be selected in $G$. The polynomial $M(x)=\sum_{j=0}^{[n / 2]}(-1)^{j} p(G, j) x^{n-2 j}$, called the matching polynomial of $G$, is closely related to the Hosoya index introduced in applications in physics and chemistry. In this work we generalize this polynomial by introducing the number of disjoint paths of length $t$, denoted by $p_{t}(G, j)$. We compare this higher-order matching polynomial with the usual one, establishing similarities and differences. Some interesting examples are given. Finally, connections between our generalized matching polynomial and hypergeometric functions are found.

\section{Introduction}

Let $G$ be a graph with $n$ vertices and let $p(G, j)$ be equal to the number of ways in which $j$ mutually nonincident edges can be selected in $G$. By definition, $p(G, 0)=1$ and clearly $p(G, 1)$ is equal to the number of edges. The Hosoya topological index $Z(G)$ is defined as follows [13]:

$$
Z(G)=\sum_{j=0}^{[n / 2]} p(G, j)
$$

This index was used by Hosoya and coworkers to correlate boiling points and other physicochemical properties of alkanes (hydrocarbons of the general formula $C_{n} H_{2 n+2}$ ) with their structure $[14,17]$. It was shown by Hosoya that for the path $P_{n}$ and the cycle $C_{n}$ graphs, his index leads to the Fibonacci and Lucas numbers, respectively.

The numbers $p(G, j)$ in the definition of the Hosoya index can be used to construct the polynomial

$$
M(G)=M(G, x)=\sum_{j=0}^{[n / 2]}(-1)^{j} p(G, j) x^{n-2 j}
$$

called the matching polynomial of the graph $G[4]$. As it turned out, this polynomial had 
been independently introduced in applications to physics and chemistry $[1,10,11,12$, 15]. Many properties of the matching polynomial have been established. For instance, the matching polynomial and the characteristic polynomial of forests are identical, its roots real, and for several classes of graphs their matching polynomials are identical to wellknown orthogonal polynomials, such as Chebyshev, Hermite, and Laguerre polynomials $[3,8]$.

On the other hand, the Hosoya index was generalized to higher-order Hosoya numbers by Randić et al. [18]. In this generalization, instead of using the numbers $p(G, j)$ which count the disjoint edges in $G$, one uses numbers $p_{t}(G, j)$ that count the number of ways of selecting $j$ mutually nonincident paths of length $t$ ( $t$-paths, for short) in $G$ (with $\left.p_{t}(G, 0):=1\right)$. This new generalized index leads to higher-order Fibonacci and Lucas numbers in the cases of linear chains and cycles, respectively. Those numbers were introduced by Staklov [20] and, independently, by Randić et al. [18, 19].

The main goal of this work is to use the numbers $p_{t}(G, j)$ to construct a polynomial, called the higher-order matching polynomial, which generalizes the standard matching polynomial, and to extend many of the results which appeared in $[3,8,9]$ to this new polynomial. For example, we establish connections between the higher-order matching polynomial and hypergeometric functions, generalizing in this way, the well-known relations with orthogonal polynomials.

The higher-order matching polynomial is a natural generalization of the usual matching or acyclic polynomial, in which the paths of length $t>1$ play the role of the edges. It is defined as

$$
M_{t}(G)=M_{t}(G, x)=\sum_{j=0}^{[n /(t+1)]}(-1)^{j} p_{t}(G, j) x^{n-(t+1) j}
$$

Actually, as we will see in Section 2, $M_{t}(G)$ is a special case of an F-polynomial of the graph $G$, introduced and developed by Farrell $[5,6,7]$. In particular, the results in Farrell's papers can be used to obtain explicit formulas of $M_{t}(G)$ for significant classes of graphs. This is done in Section 2. In Section 3 we establish connections between the higher-order matching polynomial and hypergeometric functions, generalizing in this way the wellknown relations between the matching polynomial and orthogonal polynomials.

\section{The path polynomial of a graph}

We follow the basic definitions and notations given by Farrell $[5,7]$.

Let $\mathscr{F}$ be a family of connected graphs. Let $w:=\mathscr{F} \rightarrow W$ be a function from $\mathscr{F}$ to a set of weights. An $\mathscr{F}$-cover of a graph $G$ is a spanning subgraph of $G$ in which every component belongs to $\mathscr{F}$. For each $\mathscr{F}$-cover $C$ of $G$ we define $\pi(C)=\prod_{\alpha} w(\alpha)$, where this product is taken over all components of the cover. The $F$-polynomial of $G$ is defined as

$$
F(G, w)=\sum_{C} \pi(C)
$$

where the sum is taken over all $\mathscr{F}$-covers of $G$. 
In this paper, $\mathscr{F}_{F}:=\left\{\pi_{0}, \pi_{1}, \ldots\right\}$, with $\pi_{i}$ a generic path of length $i$. The corresponding $F(G, w)$ will be called the path polynomial, written as $P(G, w)$. Further, the $t$ th order matching polynomial $M_{t}(G, x)$ will be $P(G, w)$ for the special weights $w_{1}=x, w_{t+1}=-1$ and $w_{i}=0$ otherwise, where $w_{j}$ is the weight of any path in $\mathscr{F}$ with $j$ vertices.

Next we give explicit formulas for the higher-order matching polynomials for some important classes of graphs.

THEOREM 2.1.

(1) Let $G$ be a graph with at least one t-path $Q$, and e an edge of $G$, then:

$$
M_{t}(G)=M_{t}(G-e)-\sum_{Q} M_{t}(G-Q),
$$

where the summation is taken over all paths $Q$ in $G$ containing the edge e.

(2) If $v$ is a vertex of $G$, then:

$$
M_{t}(G)=M_{t}(G-v)-\sum_{Q} M_{t}(G-Q)
$$

where the summation is taken over all paths $Q$ of length $t$ containing the vertex $v$.

Proof. (1) is a particular case of [7, Theorem 1], and (2) follows easily by applying (2.2) to all edges of $G$ incident with $v$.

From [7, Theorem 2], $P(G, w)=\prod_{i=1}^{r} P\left(H_{i}, w\right)$, where $G$ consists of $r$ components $H_{1}, H_{2}, \ldots, H_{r}$, one gets the expression $M_{t}(G)=\prod_{i=1}^{r} M\left(H_{i}\right)$.

Proposition 2.2. Let $v_{1}, \ldots, v_{n}$ be the vertices of $G$. Then

$$
\frac{d}{d x}\left[M_{t}(G)\right]=\sum_{i=1}^{n} M_{t}\left(G-v_{i}\right) .
$$

Proof. It is not difficult to prove that

$$
\frac{d}{d w_{1}} P(G, w)=\sum_{i=1}^{n} P\left(G-v_{i}, w\right) .
$$

Making the already mentioned substitution $w_{1}=x, w_{t+1}=-1$ one gets (2.4) from (2.5).

2.1. Chains $P_{n}$. In [7] Theorem 3 asserts that

$$
P\left(P_{n}, w\right)=\sum_{i=1}^{n} w_{i} P\left(P_{n-i}, w\right)
$$

which in our case reduces to

$$
M_{t}\left(P_{n}\right)=x M_{t}\left(P_{n-1}\right)-M_{t}\left(P_{n-(t+1)}\right) .
$$


1568 The higher-order matching polynomial of a graph

2.2. Circuits $C_{n}$. Theorem 11 of [7] states that

$$
P\left(C_{n}, w\right)=\sum_{r=1}^{n} r w_{r} P\left(P_{n-r}, w\right)
$$

Again, making the substitution $w_{1}=x, w_{t+1}=-1$ produces

$$
\begin{gathered}
M_{t}\left(C_{n}\right)=x M_{t}\left(P_{n-1}\right)-(t+1) M_{t}\left(P_{n-(t+1)}\right), \\
M_{t}\left(C_{n}\right)=M_{t}\left(P_{n}\right)-t M_{t}\left(P_{n-(t+1)}\right) .
\end{gathered}
$$

From the above relations it is also easy to obtain the recursive formula for $C_{n}$,

$$
M_{t}\left(C_{n}\right)=x M_{t}\left(C_{n-1}\right)-M_{t}\left(C_{n-(t+1)}\right)
$$

and the following explicit formulas for $M_{t}\left(C_{n}\right)$ and $M_{t}\left(P_{n}\right)$.

Proposition 2.3. The matching polynomials of order $t$ of $C_{n}$ and $P_{n}$ are

(1)

$$
M_{t}\left(P_{n}\right)=\sum_{j=1}^{[n /(t+1)]}(-1)^{j}\left(\begin{array}{c}
n-j t \\
j
\end{array}\right) x^{n-(t+1) j}
$$

(2)

$$
M_{t}\left(C_{n}\right)=\sum_{j=1}^{[n /(t+1)]}(-1)^{j} \frac{n}{n-j t}\left(\begin{array}{c}
n-j t \\
j
\end{array}\right) x^{n-(t+1) j} .
$$

Before we consider the cases of the complete graph $K_{n}$ and the complete bipartite graph $K_{n, m}$, we include some formulas which generalize an analogous one when $t=1$ (see [16]).

Proposition 2.4. Let $V(G)=\left\{v_{1}, v_{2}, \ldots, v_{n}\right\}$ and $E(G)=\left\{e_{1}, e_{2}, \ldots, e_{m}\right\}$ be the vertices and the edges of the graph $G$, then

$$
(t+1) \sum_{i=1}^{m} M_{t}\left(G-e_{i}, x\right)=t x \sum_{j=1}^{n} M_{t}\left(G-v_{j}, x\right)+((t+1) m-t n) M_{t}(G, x),
$$

and

(1) $(n-(t+1) k) p_{t}(G, k)=\sum_{j=1}^{n} p_{t}\left(G-v_{j}, k\right)$,

(2) $(m-t k) p_{t}(G, k)=\sum_{i=1}^{m} p_{t}\left(G-e_{i}, k\right)$

for all $1 \leq k \leq[(n-1) /(t+1)]$.

Proposition 2.5. Let $G$ be a graph, $v$ a vertex of $G$, and $e_{1}, e_{2}, \ldots, e_{d}$ the edges incident with $v$.

Then for each integer $t \geq 1$ and $l$ such that $1 \leq l \leq d-1$,

$$
\begin{aligned}
M_{t}(G)= & M_{t}\left(G-e_{1}-e_{2}-\cdots-e_{l}\right)+M_{t}\left(G-e_{l+1}-e_{l+2}-\cdots-e_{d}\right) \\
& -M_{t}\left(G-e_{1}-e_{2}-\cdots-e_{d}\right)-\sum_{Q} M_{t}(G-Q),
\end{aligned}
$$


where the last sum is taken over all such paths containing an edge $e_{i}, 1 \leq i \leq l$, and an edge $e_{j}, l+1 \leq j \leq d$.

2.3. Complete graphs $K_{n}$. In [5, Section 5] the $F$-polynomials of complete graphs are considered. Given a partition of the set $V(G)$ of vertices of the graph $G$ into $j_{i}$ sets with $i$ vertices, formula (2.2) of that section gives the contribution of each partition to $F\left(K_{n}, w\right)$

$$
B_{j}=n ! \prod_{i=1}^{n} \frac{1}{j_{i} !}\left(\frac{\phi_{i} w_{i}}{i !}\right)^{j_{i}}
$$

where $\phi_{i}$ denotes the number of spanning subgraphs of $K_{i}$, for each $1 \leq i \leq n$.

In the case of path polynomials one has $\phi_{1}=1$ and $\phi_{i}=i ! / 2$ for $2 \leq i \leq n$, hence one gets

$$
B_{j}=\frac{n !}{j_{1} !} \prod_{i=2}^{n} \frac{1}{j_{i} !} \frac{1}{2^{j_{i}}}
$$

and the corresponding term to this partition in $P\left(K_{n}, w\right)$ is, of course,

$$
B_{j} \cdot w_{1}^{j_{1}} \cdots \cdots w_{n}^{j_{n}}
$$

In the case of the higher-order matching polynomial, setting $j_{1}=n-(t+1) j, j_{t+1}=j$, the above formula turns out to be

$$
B_{j}=(-1)^{j}\left(\begin{array}{c}
n \\
(t+1) j
\end{array}\right) \frac{((t+1) j) !}{j !} \frac{1}{2^{j}}
$$

and the corresponding term will be $B_{j} x^{n-(t+1) j}$.

As far as the recursive relations for $F\left(K_{n}, w\right)$ are concerned, the following is proved in [5]:

$$
F\left(K_{n+1}, w\right)=\sum_{i=1}^{n+1}\left(\begin{array}{c}
n \\
i-1
\end{array}\right) \phi_{i} w_{i} F\left(K_{n-i+1}, w\right) .
$$

Making the substitution $M_{t}\left(K_{n}\right)$ for $F\left(K_{n}, w\right), w_{1}=x, w_{t+1}=-1, \phi_{1}=1, \phi_{t+1}=(t+$ $1) ! / 2$, results in

$$
M_{t}\left(K_{n+1}\right)=x M_{t}\left(K_{n}\right)-\left(\begin{array}{l}
n \\
t
\end{array}\right) \frac{(t+1) !}{2} M_{t}\left(K_{n-t}\right) .
$$

The explicit expression for $M_{t}\left(K_{n}\right)$ is

$$
M_{t}\left(K_{n+1}\right)=\sum_{j=0}^{[n /(t+1)]}(-1)^{j}\left(\begin{array}{c}
n \\
(t+1) j
\end{array}\right) \frac{((t+1) j) !}{j !} \frac{1}{2^{j}} x^{n-(t+1) j} .
$$


2.4. Complete bipartite graphs $K_{n, m}, n \geq m$. Here we give the general procedure to obtain $M_{t}\left(K_{n, m}\right)$ where $n$ is greater than or equal to $m$. It is convenient to consider two cases: (a) $t$ is even and (b) $t$ is odd, the latter being the easier one.

Proposition 2.6. The number $p_{t}\left(K_{n, j}, k\right)$ of the complete bipartite graph $K_{n, j}$ of $n+j$ vertices, where $j \leq n$ and $t=2 l$ is an even number, is given by

$$
p_{2 l}\left(K_{n, j}, k\right)=\frac{1}{k !} \frac{n !}{(n-k l) !} \frac{j !}{[j-k(l+1)] !} .
$$

Proof. We call $V_{n}$ and $V_{j}$ the two disjoint sets whose union is the vertex set of the graph $K_{n, j}$. Recall that we denote $l=t / 2$. Now, if we represent by $[(l, l+1)]$ a path with $l$ vertices in $V_{n}$ and $(l+1)$ vertices in $V_{j}$, there are the following possibilities for $k$ different nonincident $t$-paths in $K_{n, j}:(k-s)$ different $[(l, l+1)]$ paths and $s$ different $[(l+1, l)]$ paths, which explains the combinatorial numbers and the division by $1 /(k-s) ! s$ ! in order to avoid repetitions, in the expression

$$
p_{2 l}\left(K_{n, j}, k\right)=\left(\frac{1}{2}(l+1) ! l !\right) \sum_{s=0}^{k} \frac{1}{(k-s) ! s !} \prod_{i=0}^{k-1}\left(\begin{array}{c}
n-i l \\
l
\end{array}\right)\left(\begin{array}{c}
j-i(l+1) \\
l+1
\end{array}\right) .
$$

Now, any $(2 l+1)$ vertices determine $(l !)^{2}((l+1) / 2)$ different $2 l$-paths. This explains the factor at the beginning of the above formula for $p_{2 l}\left(K_{n, j}, k\right)$. We note that $k$ runs from 1 to $[(n+j) /(2 l+1)]$. Since

$$
\begin{gathered}
\prod_{i=0}^{k-1}\left(\begin{array}{c}
n-i l \\
l
\end{array}\right)=\frac{n !}{(l !)^{k}(n-k l) !} \\
\sum_{s=0}^{k} \frac{1}{(k-s) ! s !}=\frac{2^{k}}{k !},
\end{gathered}
$$

we obtain (2.22).

Proposition 2.7. Let $t=2 l$ be an even number. Then the following recurrence relations hold for $M_{t}\left(K_{n, m}\right), n \geq m$,

$$
\begin{aligned}
M_{2 l}\left(K_{n, m}\right)= & x M_{2 l}\left(K_{n-1, m}\right)-(l !)^{2}\left(\frac{l+1}{2}\right) \\
& \times\left[\left(\begin{array}{c}
n-1 \\
l
\end{array}\right)\left(\begin{array}{c}
m \\
l
\end{array}\right) M_{t}\left(K_{n-(l+1), m-l}\right)+\left(\begin{array}{c}
n-1 \\
l-1
\end{array}\right)\left(\begin{array}{c}
m \\
l+1
\end{array}\right) M_{2 l}\left(K_{n-l, m-(l+1)}\right)\right], \\
M_{2 l}\left(K_{n, m}\right)= & x M_{2 l}\left(K_{n, m-1}\right)-(l !)^{2}\left(\frac{l+1}{2}\right) \\
& \times\left[\left(\begin{array}{c}
n \\
l
\end{array}\right)\left(\begin{array}{c}
m-1 \\
l
\end{array}\right) M_{t}\left(K_{n-l, m-(l+1)}\right)+\left(\begin{array}{c}
n \\
l+1
\end{array}\right)\left(\begin{array}{c}
m \\
l-1
\end{array}\right) M_{2 l}\left(K_{n-(l+1), m-l}\right)\right] .
\end{aligned}
$$


Proof. Let $V_{n}$ and $V_{m}$ denote the two sets of vertices making up the vertex set of the graph $K_{n, m}$. Only (2.25) is proven here, since (2.26) follows by symmetry. We apply, again, Theorem 2.1, taking off one vertex, say $v$ of $V_{n}$. Then according to whether the different $t$ paths having $v$ as end vertex have $l$ additional points in $V_{n}$ and $l$ in $V_{m}$ or $(l-1)$ additional points in $V_{n}$ and $(l+1)$ in $V_{m}$, there are $\left(\begin{array}{c}n-1 \\ l\end{array}\right)\left(\begin{array}{c}m \\ l\end{array}\right)$ or $\left(\begin{array}{c}n-1 \\ l-1\end{array}\right)\left(\begin{array}{c}m \\ l+1\end{array}\right)$, respectively, different possibilities of choosing the other $2 l$ vertices that, together with $v$, form the $2 l$-paths. The number of different $2 l$-paths joining the $(2 l+1)$-vertices is $(l !)^{2}((l+1) / 2)$. Similarly, taking $v$ in $V_{m}$ yields (2.26).

Proposition 2.8. Let $t=2 l$ be an even number, then the matching polynomial of order $t$ of the complete bipartite graph $K_{n, n}$ is given by the following expression:

$$
M_{t}\left(K_{n, n}\right)=\sum_{k=0}^{[2 n /(t+1)]} \frac{2}{k !} \frac{(n !)^{2}}{2^{k}} \frac{(2 n-2 k l-1) !}{(n-k l) !(n-k l-1) !(2 n-k(2 l+1)) !} x^{2 n-(t+1) k} .
$$

Proof. Making the substitution $j=n$ in the formula (2.22) and making the necessary simplifications, one gets (2.27). it.

The general case of the complete bipartite graph is more complicated and we omit

Proposition 2.9. Let $t=2 l-1$ be an odd number. Then for $n \geq m$,

$$
M_{2 l-1}\left(K_{n, m}\right)=\sum_{j=0}^{[m / l]} \frac{(-1)^{j}}{j !}((j l) !)^{2}\left(\begin{array}{l}
n \\
j l
\end{array}\right)\left(\begin{array}{c}
m \\
j l
\end{array}\right) x^{n-2 l j} .
$$

Proof. We note, using the same argument as for the case $t$ even (Proposition 2.6), that the possible selections of $j$ mutually nonincident $(2 l-1)$-paths in $K_{n, m}$ are

$$
\prod_{i=0}^{j-1}\left(\begin{array}{c}
n-i l \\
l
\end{array}\right)\left(\begin{array}{c}
m-i l \\
l
\end{array}\right)=\frac{n !}{(l !)^{j}(n-j l) !} \frac{m !}{(l !)^{j}(m-j l) !} .
$$

Multiplying (2.29) by $(l !)^{2 j}$, one gets $p_{2 l-1}\left(K_{n, m}, j\right)$. Summing up such $p_{2 l-1}\left(K_{n, m}, j\right)$ and simplifying the combinatorial expression, we obtain (2.28).

Proposition 2.10. Let $t=2 l-1$ be an odd number. Then the following recurrence relations hold for $M_{2 l-1}\left(K_{n, m}\right), n \geq m$ :

$$
\begin{aligned}
& M_{2 l-1}\left(K_{n, m}\right)=x M_{2 l-1}\left(K_{n-1, m}\right)-(l !)^{2}\left(\begin{array}{c}
n-1 \\
l-1
\end{array}\right)\left(\begin{array}{c}
m \\
l
\end{array}\right) M_{2 l-1}\left(K_{n-l, m-l}\right), \\
& M_{2 l-1}\left(K_{n, m}\right)=x M_{2 l-1}\left(K_{n, m-1}\right)-(l !)^{2}\left(\begin{array}{c}
n \\
l
\end{array}\right)\left(\begin{array}{c}
m-1 \\
l-1
\end{array}\right) M_{2 l-1}\left(K_{n-l, m-l}\right) .
\end{aligned}
$$

Proof. To obtain (2.30) we apply the same criterion as in Proposition 2.7. Taking $v$ in $V_{n}$, we have $\left(\begin{array}{c}n-1 \\ l-1\end{array}\right)$ possible choices of points in $V_{n}$ and $\left(\begin{array}{c}m \\ l\end{array}\right)$ in $V_{m}$. As before, the factor $(l !)^{2}$ is the number of different $(2 l-1)$-paths joining $2 l$-vertices. By symmetry we obtain (2.31). 
1572 The higher-order matching polynomial of a graph

\section{Higher-order matching polynomial and orthogonal polynomials}

It is known that matching polynomials have connections to orthogonal polynomials [2, 8]. It is also known that those orthogonal polynomials can be expressed in terms of hypergeometric functions [2]. Here we want to explore the connections of the higher-order matching polynomial with hypergeometric functions for some simple graphs and to show that in the particular case of $t=1$ the hypergeometric functions corresponding to our higher-order matching polynomial reduce to the well-known relations.

Let $\Gamma(z)$ represent the Euler gamma function. The Pochhammer symbol, defined as $(a)_{k}=a(a+1) \cdots(a+k-1)=\Gamma(a+k) / \Gamma(a)$, is useful in the common expression of the generalized hypergeometric function

$$
{ }_{p} F_{q}(\mathbf{a} ; \mathbf{b} ; x)=\sum_{k=0}^{\infty} \frac{\left(a_{1}\right)_{k}\left(a_{2}\right)_{k} \cdots\left(a_{p}\right)_{k}}{\left(b_{1}\right)_{k}\left(b_{2}\right)_{k} \cdots\left(b_{q}\right)_{k}} \cdot \frac{1}{k !} x^{k}
$$

where $\mathbf{a}=\left(a_{1}, a_{2}, \ldots, a_{p}\right)$ and $\mathbf{b}=\left(b_{1}, b_{2}, \ldots, b_{q}\right)$.

In order to transform polynomial expressions into hypergeometric ones, it is very convenient to know the basic properties of the Pochhammer symbol, such as the following:

$$
\begin{gathered}
(a)_{-i}=\frac{(-1)^{i}}{(1-a)_{i}} \\
(a)_{k n}=\left(\frac{a}{k}\right)_{n}\left(\frac{a+1}{k}\right)_{n}\left(\frac{a+2}{k}\right)_{n} \ldots\left(\frac{a+k-1}{k}\right)_{n} k^{k n} .
\end{gathered}
$$

3.1. Paths $P_{n}$. Making use of the definition of $(a)_{k}$ and formula (3.1), we can write (2.11) as

$$
M_{t}\left(P_{n}\right)=x^{n} \sum_{j=0}^{[n /(t+1)]} \frac{(-n)_{j(t+1)}}{j !(-n)_{j t}}\left(x^{-(t+1)}\right)^{j}
$$

From (3.3) we find

$$
(-n)_{j(t+1)}=\Pi_{k=0}^{t}\left(\frac{k-n}{(t+1)_{j}}\right)(t+1)^{j(t+1)}, \quad(-n)_{j t}=\Pi_{k=0}^{t}\left(\frac{k-n}{(t)_{j}}\right)(t)^{j t} .
$$

Then, substituting (3.5) in (3.4), we obtain

$$
\begin{aligned}
M_{t}\left(P_{n}\right) & =x^{n} \sum_{j=0}^{[n /(t+1)]} \frac{\prod_{k=0}^{t}((k-n) /(t+1))_{j}}{j ! \prod_{k=0}^{t-1}((k-n) / t)_{j}}\left(\frac{(t+1)^{t+1}}{t^{t}} \frac{1}{x^{t+1}}\right)^{j} \\
& =x^{n}{ }_{t+1} F_{t}\left(\mathbf{a} ; \mathbf{b} ; \frac{(t+1)^{t+1}}{t^{t} x^{t+1}}\right),
\end{aligned}
$$

where

$$
\mathbf{a}=\left(-\frac{n}{t+1}, \frac{1-n}{t+1}, \ldots, \frac{t-n}{t+1}\right), \quad \mathbf{b}=\left(-\frac{n}{t}, \frac{1-n}{t}, \ldots, \frac{t-1-n}{t}\right)
$$


In the particular case of $t=1,(3.6)$ reduces to the known result

$$
M\left(P_{n}\right)=x_{2}^{n} F_{1}\left(-\frac{n}{2}, \frac{(1-n)}{2} ;-n ; \frac{4}{x^{2}}\right)=U_{n}\left(\frac{x}{2}\right),
$$

where $U_{n}(z)$ are the Chebyshev polynomials of the second kind [2].

3.2. Cycles $C_{n}$. As in the case of $P_{n},(2.12)$ can be written as

$M_{t}\left(C_{n}\right)=x^{n} \sum_{j=0}^{[n /(t+1)]} \frac{\prod_{k=0}^{t}((k-n) /(t+1))_{j}}{j ! \prod_{k=0}^{t-1}((k+1-n) / t)_{j}}\left(\frac{(t+1)^{t+1}}{t^{t}} \frac{1}{x^{t+1}}\right)^{j}=x^{n}{ }_{t+1} F_{t}\left(\mathbf{a} ; \mathbf{b} ; \frac{(t+1)^{t+1}}{t^{t} x^{t+1}}\right)$,

where

$$
\mathbf{a}=\left(-\frac{n}{t+1}, \frac{1-n}{t+1}, \ldots, \frac{t-n}{t+1}\right), \quad \mathbf{b}=\left(\frac{1-n}{t}, \frac{2-n}{t}, \ldots, \frac{t-n}{t}\right)
$$

In the particular case of $t=1,(3.9)$ reduces to

$$
M_{1}\left(C_{n}\right)=M\left(C_{n}\right)=x_{2}^{n} F_{1}\left(-\frac{n}{2}, \frac{1-n}{2} ; 1-n ; \frac{4}{x^{2}}\right)=2 T_{n}\left(\frac{x}{2}\right),
$$

where $T_{n}(z)$ are the Chebyshev polynomials of the first kind [2].

3.3. Complete graphs $K_{n}$. Equation (2.21) can be written as

$$
\begin{aligned}
M_{t}\left(K_{n}\right) & =x^{n} \sum_{j=0}^{[n /(t+1)]} \frac{\prod_{k=0}^{t}((k-n) /(t+1))_{j}}{j !}\left(\frac{(t+1)^{t+1}}{2(-1)^{t}} \frac{1}{x^{t+1}}\right)^{j} \\
& =x^{n}{ }_{t+1} F_{0}\left(\mathbf{a} ; \cdot ; \frac{(t+1)^{t+1}}{2(-1)^{t} x^{t+1}}\right),
\end{aligned}
$$

where

$$
\mathbf{a}=\left(\frac{-n}{t+1}, \frac{1-n}{t+1}, \ldots, \frac{t-n}{t+1}\right)
$$

In the particular case of $t=1,(3.12)$ reduces to the known result

$$
\begin{aligned}
M_{1}\left(K_{n}\right) & =M\left(K_{n}\right)=x_{2}^{n} F_{0}\left(-\frac{n}{2}, \frac{1-n}{2} ; \cdot ;-\frac{2}{x^{2}}\right) \\
& =2^{(n-1) / 2} x_{1} F_{1}\left(\frac{1-n}{2} ; \frac{3}{2} ; \frac{x^{2}}{2}\right)=2^{-n / 2} H_{n}\left(\frac{x}{\sqrt{2}}\right)=H e_{n}(x),
\end{aligned}
$$

where $H e_{n}(z)$ are the Hermite polynomials [2]. 
1574 The higher-order matching polynomial of a graph

3.3.1. Complete bipartite graphs $K_{n, m}, n \geq m$, with $t$ odd. By the same procedure used before, (2.28) can be written as

$$
\begin{aligned}
M_{t}\left(K_{n, m}\right) & =x^{n+m} \sum_{j=0}^{[2 m /(t+1)]} \frac{1}{j !} \prod_{i=0}^{(t-1) / 2}\left(\frac{2(i-n)}{t+1}\right)_{j}\left(\frac{2(i-m)}{t+1}\right)_{j}\left(-\frac{((t+1) / 2)^{t+1}}{x^{t+1}}\right)^{j} \\
& =x^{n+m}{ }_{t+1} F_{0}\left(\mathbf{a} ; \cdot ;-\frac{((t+1) / 2)^{t+1}}{x^{t+1}}\right),
\end{aligned}
$$

where

$$
\begin{aligned}
\mathbf{a}=( & -\frac{2 n}{t+1}, \frac{2(1-n)}{t+1}, \ldots, \frac{2(((t-1) / 2)-n)}{t+1}, \\
& \left.-\frac{2 m}{t+1}, \frac{2(1-m)}{t+1}, \ldots, \frac{2(((t-1) / 2)-m)}{t+1}\right) .
\end{aligned}
$$

In the particular case of $t=1,(3.15)$ reduces to

$$
\begin{aligned}
M_{1}\left(K_{n, m}\right) & =M\left(K_{n, m}\right)=x^{n+m}{ }_{2} F_{0}\left(-n,-m ; \cdot ;-\frac{1}{x^{2}}\right) \\
& =x^{n-m} U\left(-m ; 1-m+n ; x^{2}\right)=(-1)^{m} x^{n-m} m ! L_{m}^{(n-m)}\left(x^{2}\right),
\end{aligned}
$$

where $U(a, b, z)$ is the confluent hypergeometric function of the second kind and $L_{\alpha}^{(\beta)}(z)$ are the generalized Laguerre polynomials [2].

When $n=m$, (3.17) yields

$$
M_{1}\left(K_{n, n}\right)=M\left(K_{n, n}\right)=x_{2}^{2 n} F_{0}\left(-n,-n ; \cdot ;-\frac{1}{x^{2}}\right)=U\left(-n ; 1 ; x^{2}\right)=(-1)^{n} n ! L_{n}\left(x^{2}\right),
$$

where $L_{n}(x)=L_{n}^{(0)}(x)$ is a Laguerre polynomial [2].

3.4. Complete bipartite graphs $K_{n, n}$ with $t$ even. Equation (2.27) can be written as

$$
\begin{aligned}
M_{t}\left(K_{n, n}\right)= & x^{2 n} \sum_{k=0}^{[2 n /(t+1)]} \frac{1}{k !} \frac{\prod_{j=0}^{(t / 2)-1}(2(j-n) / t)_{k}}{\prod_{j=0}^{t-1}((j+1-2 n) / t)_{k}} \prod_{j=0}^{(t / 2)-1}\left(\frac{2(j-n+1)}{t}\right)_{k} \\
& \times \prod_{j=0}^{t}\left(\frac{j-2 n}{1+t}\right)_{k}\left[-\left(\frac{1+t}{2 x}\right)^{1+t}\right]^{k}=x^{2 n}{ }_{2 t+1} F_{t}(\mathbf{a} ; \mathbf{b} ; z),
\end{aligned}
$$

where

$$
\begin{gathered}
\mathbf{a}=\left(-\frac{2 n}{t}, \ldots, 1-\frac{2(1+n)}{t}, \frac{2(1-n)}{t}, \ldots, 1-\frac{2 n}{t},-\frac{2 n}{1+t}, \ldots, \frac{t-2 n}{1+t}\right), \\
\mathbf{b}=\left(\frac{1-2 n}{t}, \ldots, 1-\frac{2 n}{t}\right), \\
z=-\left(\frac{1+t}{2 x}\right)^{1+t} .
\end{gathered}
$$




\section{Acknowledgments}

We are grateful to Professor M. Randić for his original suggestion of the possible use of the higher-order Hosoya index to construct a new higher-order matching polynomial. We thank Professor I. Gutman for useful comments and suggestions and also the anonymous referee for his careful reading of the manuscript. M. Estrada thanks the CIEN, Universidad de Antioquia, for partial support of his research. This work was partially supported by Consejo de Desarrollo Científico, Humanístico y Tecnológico (CDCHT), Universidad de Los Andes.

\section{References}

[1] J. I. Aihara, A new definition of Dewar-type resonance energies, J. Amer. Chem. Soc. 98 (1976), no. $10,2750-2758$.

[2] G. E. Andrews, R. Askey, and R. Roy, Special Functions, Encyclopedia of Mathematics and Its Applications, edited by G. C. Rota, vol. 71, Cambridge University Press, Cambridge, 1999.

[3] D. M. Cvetković, M. Doob, I. Gutman, and A. Torgašev, Recent Results in the Theory of Graph Spectra, Annals of Discrete Mathematics, vol. 36, North-Holland, Amsterdam, 1988.

[4] E. J. Farrell, An introduction to matching polynomials, J. Combin. Theory Ser. B 27 (1979), no. 1, 75-86.

[5] _ On a general class of graph polynomials, J. Combin. Theory Ser. B 26 (1979), no. 1, $111-122$.

[6] On a class of polynomials associated with the paths in a graph and its application to minimum nodes disjoint path coverings of graphs, Int. J. Math. Math. Sci. 6 (1983), no. 4, $715-726$.

[7] _ Path decompositions of chains and circuits, Int. J. Math. Math. Sci. 6 (1983), no. 3, 521-533.

[8] C. D. Godsil and I. Gutman, On the matching polynomial of a graph, Algebraic Methods in Graph Theory, Vol. I, II (Szeged, 1978), Colloq. Math. Soc. János Bolyai, vol. 25, NorthHolland, Amsterdam, 1981, pp. 241-249.

[9] I. Gutman, The acyclic polynomial of a graph, Publ. Inst. Math. (Beograd) (N.S.) 22(36) (1977), 63-69.

[10] I. Gutman, M. Milun, and N. Trinajstić, Nonparametric resonance energies of arbitrary conjugated systems, J. Amer. Chem. Soc. 99 (1977), no. 6, 1692-1704.

[11] O. J. Heilmann and E. H. Lieb, Monomers and dimers, Phys. Rev. Lett. 24 (1970), no. 25, 14121414.

[12] Theory of monomer-dimer systems, Comm. Math. Phys. 25 (1972), 190-232.

[13] H. Hosoya, Topological index. A newly proposed quantity characterizing the topological nature of structural isomers of saturated hydrocarbons, Bull. Chem. Soc. Jpn 44 (1971), 2332-2339.

[14] Topological index and thermodynamics properties. I. Empirical rules of the boiling point of saturated hydrocarbons, Bull. Chem. Soc. Jpn 45 (1972), 3415-3422.

[15] H. Kunz, Location of the zeros of the partition function for some classical lattice systems, Phys. Lett. A 32 (1970), 311-312.

[16] J. J. Mira, Polinomios acíclicos o de pareo y otros polinomios asociados a grafos, Tesis de Maestría, Facultad de Ciencias, Departamento de Matemáticas, Universidad de Antioquia, Medellín, 2003.

[17] H. Narumi and H. Hosoya, Topological index and thermodynamics properties. II. Analysis of the topological factors on the absolute entropy of acyclic saturated hydrocarbons, Bull. Chem. Soc. Jpn 53 (1980), 1228-1237. 


\section{The higher-order matching polynomial of a graph}

[18] M. Randić, D. A. Morales, and O. Araujo, Higher-order Fibonacci numbers, J. Math. Chem. 20 (1996), no. 1-2, 79-94.

[19] Higher-order Lucas numbers, unpublished.

[20] A. P. Stakhov, Golden Ratio Codes, Cybernetics, Radio i Svyaz', Moscow, 1984.

Oswaldo Araujo: Departamento de Matemáticas, Facultad de Ciencias, Universidad de Los Andes, Mérida 5101, Venezuela

E-mail address: araujo@ula.ve

Mario Estrada: ICIMAF, La Habana, Cuba

E-mail address: mestrada@icmf.inf.cu

Daniel A. Morales: Facultad de Ciencias, Universidad de Los Andes, Apartado Postal A61, La Hechicera, Mérida 5101, Venezuela

E-mail address: danoltab@ula.ve

Juan Rada: Departamento de Matemáticas, Facultad de Ciencias, Universidad de Los Andes, Mérida 5101, Venezuela

E-mail address: juanrada@ula.ve 


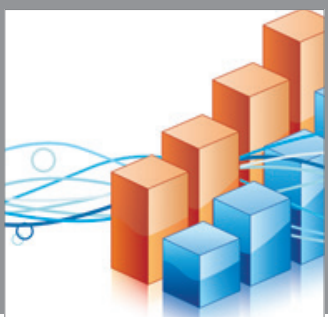

Advances in

Operations Research

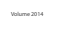

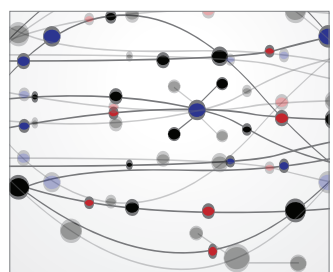

\section{The Scientific} World Journal
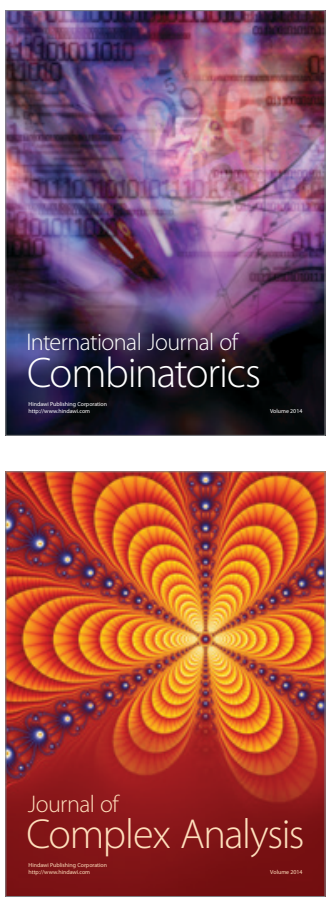

International Journal of

Mathematics and

Mathematical

Sciences
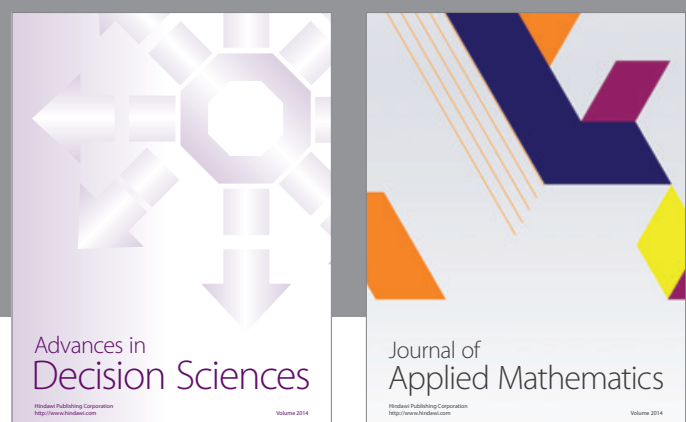

Journal of

Applied Mathematics
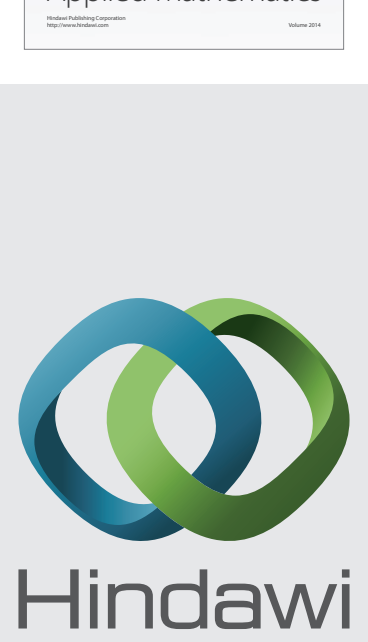

Submit your manuscripts at http://www.hindawi.com
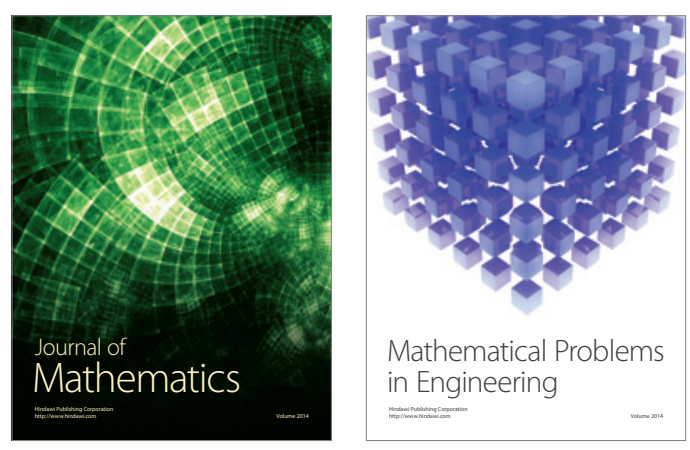

Mathematical Problems in Engineering
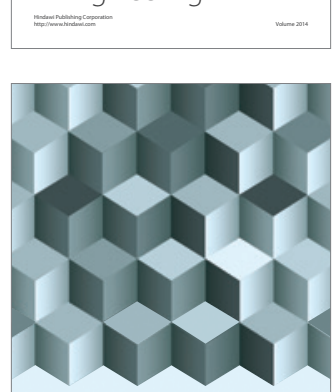

Journal of

Function Spaces
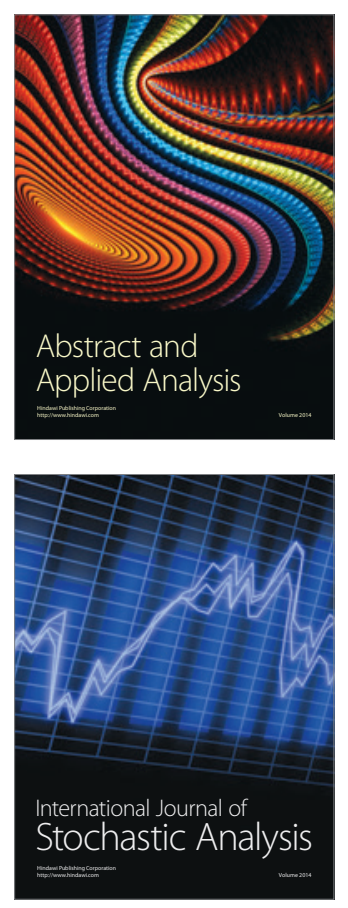

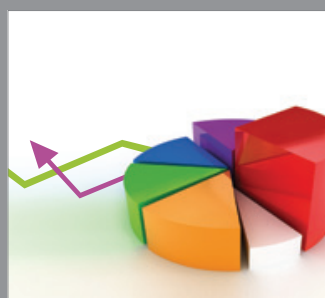

ournal of

Probability and Statistics

Promensencen
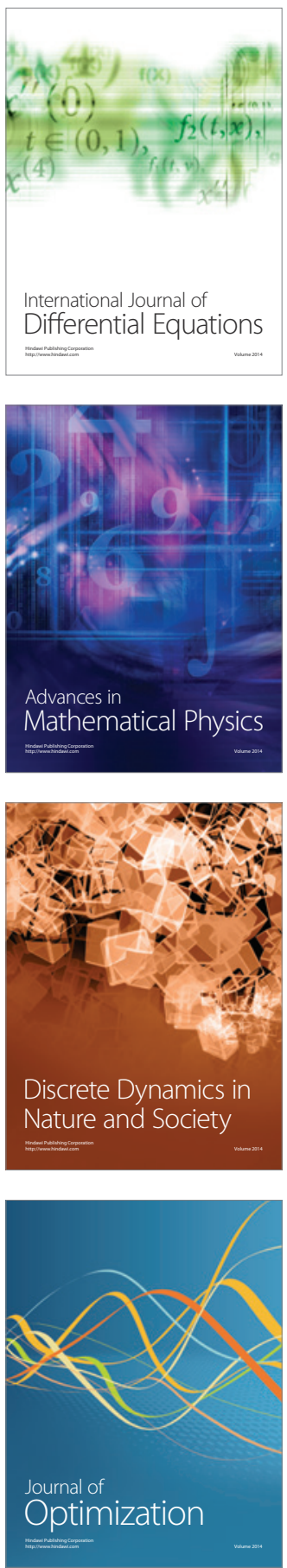